\title{
Inmigración y Preferencias por la Redistribución en América Latina
}

\author{
Alumno: Julián Martinez-Correa* \\ Director: Leonardo Gasparini
}

Mayo, 2020

\begin{abstract}
Resumen
Este trabajo analiza el efecto de la inmigración en las preferencias por la redistribución en América Latina. Para ello, sigo una doble estrategia de investigación: un análisis transversal con censos armonizados y un enfoque de efectos fijos con encuestas de hogares, ambos unidos con datos de actitudinales para el período 2008-2016. Los resultados sugieren una relación negativa entre el porcentaje de inmigrantes a nivel provincial y el apoyo a las políticas redistributivas. El caso específico de la migración venezolana a Colombia muestra resultados en la misma dirección. Este efecto anti-distributivo es mayor entre las personas que se consideran ideológicamente de derecha, son altamente calificadas y tienen altos ingresos, y no trabajan en el sector público. Además, al considerar las características de los inmigrantes, encuentro que el efecto se explica principalmente por la inmigración de baja calificación y de países fronterizos.
\end{abstract}

Códigos JEL: D63, O15, N36.

Palabras Clave: Migración, Desigualdad, Redistribución, América Latina

\footnotetext{
${ }^{*}$ Universidad Nacional de La Plata - Facultad de Ciencias Económicas, Calle 6 entre 47 y 48, La Plata 1900, Argentina. E-mail: julian.martinezcorrea@gmail.com.
} 


\section{Introducción}

América Latina es una de las regiones más desiguales del mundo y las políticas redistributivas han contribuido a mitigar este problema socio-económico. Sin embargo, desde 2010, el porcentaje de individuos que muestra un apoyo significativo a las políticas fuertes para reducir la desigualdad de ingresos ha disminuido más del $17 \%$ (Ver Figura A1). Al mismo tiempo, la inmigración aumentó considerablemente, principalmente debido a las corrientes masivas de migrantes impulsadas por crisis económicas y sociales en sus países de origen. Las percepciones acerca de estos inmigrantes y los prejuicios per se contra ellos pueden estar afectando las preferencias por la redistribución de la población (Alesina y Stantcheva, 2020).

En este documento analizo empíricamente la relación entre la inmigración y las preferencias de redistribución en América Latina. Utilizo dos estrategias diferentes. En primer lugar, construyo un novedoso conjunto de datos del porcentaje de inmigración a nivel de provincia/estado/departamento ${ }^{1}$ de censos harmonizados (IPUMS International) unidos con las encuestas actitudinales bi-anuales de Latin American Public Opinion Project (LAPOP) para 20082016. Esto me permite realizar un análisis de corte transversal con 18 países y 290 provincias.

Los resultados muestran una relación negativa entre la proporción de inmigrantes de la provincia y las preferencias por la redistribución de la población residente. Estos resultados son estables entre distintas especificaciones, robustos a formas alternativas de medir las preferencias de redistribución, a excluir las ciudades capitales, a restricciones en la muestra y un ejercicio de placebo.

La información demográfica y socio-económica detallada disponible en los datos de censo y de LAPOP me permite analizar efectos heterogéneos tanto del lado del encuestado como del de la inmigración. Específicamente, encuentro que el efecto de la inmigración en contra de la redistribución es mayor para los encuestados altamente calificados y de altos ingresos, aquellos que se consideran a sí mismos ideológicamente de derecha y empleados privados. Además, al considerar las características de los inmigrantes, encuentro que el efecto se explica completamente por la inmigración de países fronterizos y de baja calificación.

Como segunda estrategia, utilizo datos de inmigración de las encuestas de hogares y aprovecho la estructura de datos del panel para estimar un modelo de efectos fijos. Al unir cada encuesta de hogares con la respectiva onda de LAPOP, exploto la variación de corto plazo de los inmigrantes y las actitudes en el tiempo y entre provincias. De esta forma, esta especificación se centra en los efectos a corto plazo.

Las estimaciones de este modelo con efectos fijos muestran de nuevo un claro efecto negativo en las preferencias por la redistribución frente a la mayor exposición a los inmigrantes. Los efectos heterogéneos muestran resultados coherentes con los del análisis de corte transversal: más fuertes en los individuos ideológicamente de derecha y altamente calificados y procedentes principalmente de inmigrantes de baja calificación y bajos ingresos.

Finalmente, exploto la reciente inmigración venezolana en Colombia con los datos de los

\footnotetext{
${ }^{1}$ En lo sucesivo, me refiero a estados, departamentos y provincias - dependiendo de la división política de los países - simplemente como provincias.
} 
ingresos al país. Las estimaciones de un modelo de efectos fijos para este caso específico muestran resultados confirmatorios.

Lo más cercano a este trabajo es un creciente cuerpo de literatura para los Estados Unidos y Europa que muestra que la inmigración reduce el apoyo por las políticas redistributivas (Alesina et al., 2019; Dahlberg et al., 2012; Razin et al., 2002; Tabellini, 2018, 2019). De hecho, dados los recientes flujos migratorios en América Latina, me baso en Alesina et al. (2019) y trato de responder a la pregunta sobre la inmigración y el apoyo a la redistribución en la región. Contrariamente a la literatura existente -que se ha centrado en la inmigración procedente de países en desarrollo a países desarrollados-, analizo el caso en que la inmigración procede principalmente de países de la misma región y, por lo tanto, con antecedentes culturales similares. Contrariamente a lo que podría esperarse, en el caso de América Latina sigue habiendo un gran efecto negativo en las preferencias de redistribución. Hasta donde conozco, no hay pruebas de esta relación en los países en desarrollo. Por último, también contribuyo construyendo una novedosa base de datos del porcentaje de inmigración a nivel de provincia para la mayoría de los países de América Latina a lo largo del tiempo.

Este trabajo también está relacionado con la literatura que estudia los efectos de la inmigración en el surgimiento de candidatos políticos de extrema derecha (Barone et al., 2016; Becker et al., 2016; Brunner y Kuhn, 2018; Edo et al., 2019; Dinas et al., 2019; Dustmann et al., 2019; Halla et al., 2017; Harmon, 2018; Otto y Steinhardt, 2014; Steinmayr, 2020). En el caso de Colombia, Rozo y Vargas (2019) muestran que los flujos migratorios venezolanos desplazan los votos hacia las ideologías de derecha. Mis resultados arrojan algo de luz sobre el mecanismo subyacente a este impacto electoral encontrados en la literatura: un aumento de la inmigración poco calificada reduce las preferencias por las políticas redistributivas entre los votantes y, a través de este canal, puede aumentar las posibilidades de que los candidatos de extrema derecha ganen las elecciones.

El resto del documento está organizado de la siguiente manera: En la sección 2 se describen las fuentes de datos. En las secciones 3 y 4 se presentan las estrategias empíricas y los resultados del análisis de corte transversal y la estimación de efectos fijos, respectivamente. La sección 5 analiza el caso de Colombia. Finalmente, la sección 6 concluye.

\section{Datos}

En esta sección, describo las fuentes de datos y la forma en que las uno.

\subsection{Datos Actitudinales}

Para analizar el apoyo a las políticas de redistribución utilizo las encuestas de LAPOP para el período 2008-2016. La encuesta se realiza cada dos años y es representativa a nivel nacional. Contiene información socio-económica y de actitudes a nivel individual para casi todos los países de la región. Restrinjo la muestra a las personas de 18 a 65 años de edad.

Para medir las preferencias en materia de redistribución utilizo una pregunta de la encuesta 
que capta el grado de acuerdo con la intervención del Estado para reducir la desigualdad de ingresos. Más específicamente, los encuestados responden en una escala del 1 al 7 , donde el 1 indica "Totalmente en desacuerdo" y el 7 "Totalmente de acuerdo", la siguiente pregunta: "El gobierno (del país) debe aplicar políticas firmes para reducir la desigualdad de ingresos entre los ricos y los pobres. ¿En qué medida está de acuerdo o en desacuerdo con esta afirmación?".

Como robustez, también considero preguntas alternativas relacionadas con las dimensiones de bienestar, empleo y salud, y calculo un índice que mide las preferencias de redistribución mediante la aplicación del Análisis de Componentes Principales (PCA) (Véase la Tabla B1 en el apéndice B para obtener información detallada sobre las preguntas de la encuesta). Todas las variables están estandarizadas en aras de la comparabilidad ${ }^{2} 3$

\subsection{Datos Inmigración}

Utilizo dos fuentes de información para obtener la proporción de inmigrantes a nivel de provincia. La definición de inmigrante se deriva del lugar de nacimiento (es decir, haber nacido en un país extranjero).

Datos Censo. Utilizo datos de censo provenientes de IPUMS International para construir una novedosa base de datos con la proporción de inmigrantes en la población a nivel de provincia para 18 países de América Latina. La mayoría de los países tienen uno o dos censos disponibles desde el año $2000^{4}$. Esta fuente también tiene información sobre las características de los inmigrantes (nivel de educación, país de origen, etc.).

Utilizo esta fuente de información para el análisis de corte transversal. Como los datos de LAPOP contienen información precisa sobre la residencia de los encuestados, uno los datos a nivel individual con la proporción de inmigrantes a nivel de provincia. Más concretamente, cada encuesta anual de países del LAPOP se une con el censo inmediatamente anterior disponible. La muestra final consta de 87.994 individuos para 18 países y 290 provincias entre 2008 y 2016.

Encuestas de Hogares. Utilizo los datos de la Socio-Economic Database for Latin America and the Caribbean (SEDLAC) para construir una base de datos novedosa, única y armonizada de las proporciones de inmigrantes a nivel provincial para la mayoría de los países de América Latina y no solo los años con censo. Esta base de datos es construida por el CEDLAS y el Banco Mundial llevando a cabo un proceso de armonización de las encuestas nacionales de hogares y, por lo tanto, haciéndolas comparables entre países. También cuenta con información sobre las características de los inmigrantes (nivel de educación, nivel de ingresos, etc.).

Estos datos los utilizo para la estimación del modelo de efectos fijos. En este caso, la unión de las bases es más directa: uno cada onda LAPOP con los datos de inmigración a nivel de

\footnotetext{
${ }^{2}$ El Apéndice B también provee estadísticas descriptivas de la muestra.

${ }^{3}$ Una de las limitaciones de mi trabajo es que no puedo distinguir entre inmigrantes y nativos en los encuestados de LAPOP. Sin embargo, el Latinobarómetro, una encuesta similar, que cuenta con información sobre el país de nacimiento, muestra que en 2010 los inmigrantes son una proporción muy pequeña de los encuestados (en promedio 2,3\%) que difícilmente esté impulsando los resultados. Además, como muestro más adelante, los resultados provienen principalmente de individuos de altos ingresos y alta calificación, que no son las características comunes de los inmigrantes.

${ }^{4}$ Ver Tabla C1 del Apéndice C para detalles de las fuentes de información
} 
provincia de las encuestas de hogares del año correspondiente. Estos datos me permiten analizar 11 países y 210 provincias de América Latina ${ }^{5}$.

Una desventaja de esta fuente de información es que los datos sobre los inmigrantes son más ruidosos que con los datos del censo ${ }^{6}$. Sin embargo, en el Apéndice C, la figura C1 muestra que existe una fuerte correlación entre ambas fuentes de información ${ }^{7}$. Esto sugiere que las encuestas de hogares reflejan la proporción real de inmigrantes.

\subsection{Datos Socio-Demográficos}

También me baso en las encuestas de hogares para construir variables socio-demográficas a nivel de la provincia. De esta fuente obtengo la población total, la tasa de desempleo, el ingreso medio per cápita familiar y la proporción de población rural.

\section{Análisis de corte-transversal}

\subsection{Estrategia empírica}

Para analizar la relación entre la inmigración y las preferencias de redistribución estimo el siguiente modelo de regresión:

$$
R_{i p r c t}=\alpha+\beta M_{\text {prct }}+X_{\text {iprct }} \theta+Z_{\text {prct }} \phi+\lambda_{\text {crt }}+\epsilon_{\text {iprct }}
$$

donde $R_{i p r c t}$ es el apoyo a la reducción en la desigualdad de ingresos del individuo $i ; M_{\text {prct }}$ es la proporción de inmigrantes en la provincia $p$ en la región $r$ (in $\operatorname{logs})^{8} ; X_{i p r c t}$ es un vector de controles individuales (años de educación, edad, sexo, edad*sexo, religión, estado de empleo, ideología y escala de ingresos) y $Z_{p r c t}$ es un vector de controles provinciales (el log de la población, tasa de desempleo, media del ingreso per cápita familiar y la proporción de población rural); $\lambda_{c r t}$ son efectos fijos a nivel país-región-año. Los errores estándar están clustereados a nivel provincia-año para tener en cuenta la posible correlación dentro de este nivel.

Esta especificación explota la variación de los inmigrantes dentro de una región-año. En pocas palabras, dentro de una región-año compara el apoyo a las políticas de redistribución entre las provincias con una proporción relativamente alta de inmigrantes con las que tienen una proporción relativamente baja.

La literatura de migración ha discutido mucho la posible endogeneidad de la elección del lugar de residencia de los migrantes (ver, por ejemplo, Altonji y Card, 1991; Sasin y McKenzie, 2007). En mi caso, la principal preocupación es que los factores no observados pueden afectar tanto a la inmigración como a las preferencias de redistribución. Por ejemplo, si los migrantes

\footnotetext{
${ }^{5}$ Ver el Apéndice C para detalles sobre los países y los años disponibles.

${ }^{6}$ Restrinjo la muestra a las provincias con un número positivo de inmigrantes para evitar cambios extremadamente grandes en la proporción de inmigrantes.

${ }^{7}$ El coeficiente de una regresión entre las dos variables con efectos fijos a nivel país es de 0,87 .

${ }^{8}$ Los resultados son robustos al utilizar una especificación cuadrática para la proporción de inmigrantes.
} 
fluyen hacia destinos con sistemas de bienestar más generosos (los llamados "imanes del bienestar"), que pueden ser el resultado de las preferencias de la población, podemos esperar un sesgo positivo (Borjas, 1999). Esta fuente de endogeneidad podría sesgar los resultados hacia cero.

Por otra parte, el crecimiento económico de la provincia y las perspectivas de movilidad ascendente pueden estar influyendo en las decisiones de localización de los inmigrantes. Incluimos algunos controles provinciales como la tasa de desempleo, la media del ingreso per cápita familiar y la proporción de la población rural para tratar de resolver este problema. También realizamos algunos controles de robustez como la exclusión de las ciudades capitales. El enfoque de los efectos fijos con encuestas de hogares también trata de abordar este potencial problema.

\subsection{Resultados}

Resultados principales. La Tabla 1 muestra las estimaciones de la ecuación (1) para el apoyo a la reducción de las diferencias de ingresos. Las columnas incorporan controles de forma secuencial: efectos fijos de país-región-año, controles provinciales, controles individuales y el control de la ideología individual. En todas las especificaciones, encuentro que, en promedio, hay una asociación negativa entre la proporción de inmigrantes de la provincia y el apoyo a la redistribución de la población residente en la provincia. Más específicamente, con el conjunto completo de controles obtengo que un aumento de 1 punto en el log de la proporción de inmigrantes reduce el apoyo a las políticas redistributivas en más de 0,026 desvíos estándar ${ }^{9}$.

Para obtener un orden de magnitud del coeficiente, vale notar que un aumento del p25 al p75 en la escala de ideología (de un valor de 3 a 7), reduce el apoyo a la redistribución en un $7,3 \%$ de la desviación estándar de las preferencias. En comparación, un aumento análogo en el logaritmo de la proporción de inmigrantes $(1,68)$ tiene un cambio asociado en las actitudes que es casi un $60 \%$ tan grande como el cambio en las preferencias asociado con el aumento de la escala de ideología (es decir, un 4,3\% del sd de las preferencias). Por último, en términos de la variación entre provincias, un desvío estándar en la proporción de inmigrantes reduce el apoyo a la redistribución en un 10,2\% del desvío estándar de preferencias entre provincias.

Efectos heterogéneos. Para comprender mejor los mecanismos que subyacen a esta relación negativa, examino algunos efectos heterogéneos en relación con las características de los individuos encuestados y el tipo de inmigración.

En primer lugar, analizo si el efecto anti-redistribución depende de la posición ideológica del encuestado. La Tabla 2 muestra que el efecto negativo de la inmigración en las preferencias por la redistribución está impulsado principalmente por individuos de auto-ubicados en la extrema derecha.

Otra dimensión relevante refiere al nivel de calificación de los encuestados y su interacción con la reacción a la inmigración. La Tabla 3 muestra que el efecto parece ser ligeramente mayor para los encuestados de alta calificación que para los de baja calificación.

\footnotetext{
${ }^{9}$ En otras palabras, un aumento de un sd en la proporción de inmigrantes $(1,47)$ reduce el apoyo a las políticas de redistribución en un 3,8\% de la desviación estándar de las preferencias.
} 
Tabla 1: Principales resultados: efecto de la inmigración en el apoyo a la reducción de las diferencias de ingresos

\begin{tabular}{|c|c|c|c|c|}
\hline & (I) & (II) & (III) & $(\mathrm{IV})$ \\
\hline Share inmigrantes (log) & $\begin{array}{c}-0.0235^{* *} \\
(0.0110)\end{array}$ & $\begin{array}{c}-0.0261^{* *} \\
(0.0116)\end{array}$ & $\begin{array}{c}-0.0256^{* *} \\
(0.0116)\end{array}$ & $\begin{array}{c}-0.0261^{* *} \\
(0.0115)\end{array}$ \\
\hline Observaciones & 87,944 & 87,944 & 87,944 & 87,944 \\
\hline R-cuadrado & 0.088 & 0.088 & 0.090 & 0.094 \\
\hline EF país-región-año & $\mathrm{Si}$ & $\mathrm{Si}$ & $\mathrm{Si}$ & $\mathrm{Si}$ \\
\hline Contorles provinciales & No & $\mathrm{Si}$ & $\mathrm{Si}$ & $\mathrm{Si}$ \\
\hline Controles individuales & No & No & $\mathrm{Si}$ & $\mathrm{Si}$ \\
\hline Control ideología & No & No & No & $\mathrm{Si}$ \\
\hline \multicolumn{5}{|c|}{$\begin{array}{l}\text { *** Significatividad al } 1 \% \text {; }{ }^{*} \text { significatividad al } 5 \% ;{ }^{*} \text { significatividad al } 10 \% \text {. } \\
\text { Notas: La variable dependiente mide el apoyo a la reducción de las diferencias de ingresos } \\
\text { y se basa en el grado de acuerdo con la siguiente afirmación "El gobierno (país) debe } \\
\text { aplicar políticas fuertes para reducir la desigualdad de ingresos entre los ricos y los } \\
\text { pobres". Está estandarizada (z-score). Los controles provinciales incluyen el log de la } \\
\text { población, la tasa de desempleo, la media del ingreso per cápita familar y la proporción } \\
\text { de la población rural; los controles individuales incluyen los años de educación, la edad, } \\
\text { el sexo, edad*sexo, la religión, la situación laboral y la escala de ingresos. El control } \\
\text { ideológico considera la forma en que el encuestado se sitúa en una escala del } 1 \text { al 10, } \\
\text { en la que el } 1 \text { es de izquierda y el } 10 \text { de derecha. Errores estándar clustereados a nivel } \\
\text { provincia-año entre paréntesis. }\end{array}$} \\
\hline
\end{tabular}

Tabla 2: Efectos heterogéneos: Ideología

\begin{tabular}{lcccc}
\hline & $(\mathrm{I})$ & $(\mathrm{II})$ & $(\mathrm{III})$ & $(\mathrm{IV})$ \\
\hline & & & & \\
Share inmigrantes $(\log )$ & 0.00349 & 0.000934 & 0.00201 & 0.000649 \\
& $(0.0151)$ & $(0.0155)$ & $(0.0154)$ & $(0.0152)$ \\
Share inmigrantes $(\log )$ x extrema-derecha & $-0.0312^{* * *}$ & $-0.0311^{* * *}$ & $-0.0317^{* * *}$ & $-0.0305^{* * *}$ \\
& $(0.0118)$ & $(0.0118)$ & $(0.0117)$ & $(0.0115)$ \\
& & & \\
Observaciones & 87,944 & 87,944 & 87,944 & 87,944 \\
R-cuadrado & 0.089 & 0.090 & 0.092 & 0.094 \\
\hline EF país-región-año & Si & Si & Si & Si \\
Controles provinciales & No & Si & Si & Si \\
Controles individuales & No & No & Si & Si \\
Control ideología & No & No & No & Si \\
\hline *** Significatividad al 1\%; ** significatividad al 5\%; *ignificatividad al 10\%. & & \\
Notas: La variable extrema-derecha es una variable binaria que indica la auto-selección del encuestado en los valores \\
8, 9 o 10 de la escala izquierda-derecha. Errores estándar clustereados a nivel provincia-año entre paréntesis.
\end{tabular}


Tabla 3: Efectos heterogéneos: Nivel de calificación

\begin{tabular}{lcccc}
\hline & $(\mathrm{I})$ & $(\mathrm{II})$ & $(\mathrm{III})$ & $(\mathrm{IV})$ \\
\hline & & & & \\
Share inmigrantes $(\log )$ & -0.0157 & -0.0185 & -0.0188 & $-0.0195^{*}$ \\
& $(0.0113)$ & $(0.0119)$ & $(0.0119)$ & $(0.0119)$ \\
Share inmigrantes $(\log ) \mathrm{x}$ & $-0.0248^{* * *}$ & $-0.0244^{* * *}$ & $-0.0213^{* * *}$ & $-0.0208^{* * *}$ \\
alta-calificación & $(0.00692)$ & $(0.00690)$ & $(0.00702)$ & $(0.00703)$ \\
& & & & \\
& 87,944 & 87,944 & 87,944 & 87,944 \\
Observaciones & 0.088 & 0.089 & 0.091 & 0.094 \\
R-cuadrado & $\mathrm{Si}$ & $\mathrm{Si}$ & $\mathrm{Si}$ & $\mathrm{Si}$ \\
\hline EF país-región-año & $\mathrm{No}$ & $\mathrm{Si}$ & $\mathrm{Si}$ & $\mathrm{Si}$ \\
Controles provinciales & $\mathrm{No}$ & $\mathrm{No}$ & $\mathrm{Si}$ & $\mathrm{Si}$ \\
Controles individuales & $\mathrm{No}$ & $\mathrm{No}$ & $\mathrm{No}$ & $\mathrm{Si}$ \\
Control ideología & No & No & \\
\hline *** Significatividad al 1\%; ** significatividad al 5\%; significatividad al 10\%. & \\
Notas: La variable alta-calificación identifica individuos con educación terciaria o más. Errores \\
estándar clustereados a nivel provincia-año entre paréntesis.
\end{tabular}

El nivel de ingresos del encuestado puede ser otro determinante importante del efecto antiredistributivo de la inmigración. En la Tabla 4 encuentro que el efecto de la inmigración en las preferencias de redistribución es más pronunciado en los individuos que están en la cola derecha de la distribución de ingresos. De hecho, cuanto más altos son los ingresos de los encuestados, menor es su apoyo a las políticas redistributivas como resultado de la inmigración. Este resultado es coherente con el hecho de que es probable que las personas más ricas paguen diferentes impuestos directos que pueden afectar a su percepción del gasto público y la redistribución cuando perciben a los inmigrantes como beneficiarios.

La Tabla 5 sugiere que la asociación negativa es más fuerte para los trabajadores privados. Los empleados privados podrían ser más conscientes de la presencia de inmigrantes, ya que los inmigrantes suelen trabajar en el sector privado, pero también puede ser el caso de que los auto-seleccionados empleados públicos tengan menos prejuicios contra los inmigrantes.

También exploro si el nivel de calificación de los inmigrantes tiene un efecto diferencial en las actitudes de los expuestos a la inmigración. La Tabla 6 muestra que el efecto anti-redistributivo se explica por la inmigración de baja cualificación, mientras que para la proporción de inmigrantes de alta calificación se obtiene un coeficiente no significativo. Este resultado puede explicarse porque los inmigrantes de baja cualificación tienen más probabilidades de beneficiarse del sistema de bienestar social que los inmigrantes de alta calificación o porque hay un mayor sesgo en su contra.

Adicionalmente, considero el origen de los inmigrantes en la Tabla 7. Encuentro que el efecto anti-redistribución es impulsado por los inmigrantes provenientes de países fronterizos.

Por último, exploro los efectos heterogéneos en los países de destino. La Tabla 8 muestra 
Tabla 4: Efectos heterogéneos: Nivel de ingresos

\begin{tabular}{lcccc}
\hline & $(\mathrm{I})$ & $(\mathrm{II})$ & $(\mathrm{III})$ & $(\mathrm{IV})$ \\
\hline & & & & \\
Share inmigrantes $(\log )$ & $-0.0213^{*}$ & $-0.0244^{* *}$ & $-0.0242^{* *}$ & $-0.0248^{* *}$ \\
& $(0.0110)$ & $(0.0116)$ & $(0.0116)$ & $(0.0116)$ \\
Share inmigrantes $(\log ) \mathrm{x}$ & $-0.0342^{* *}$ & $-0.0341^{* *}$ & $-0.0297^{* *}$ & $-0.0285^{* *}$ \\
alto-ingreso & $(0.0136)$ & $(0.0136)$ & $(0.0136)$ & $(0.0134)$ \\
& & & & \\
Observaciones & 87,944 & 87,944 & 87,944 & 87,944 \\
R-cuadrado & 0.088 & 0.088 & 0.091 & 0.094 \\
\hline EF país-región-año & $\mathrm{Si}$ & $\mathrm{Si}$ & $\mathrm{Si}$ & $\mathrm{Si}$ \\
Controles provinciales & $\mathrm{No}$ & $\mathrm{Si}$ & $\mathrm{Si}$ & $\mathrm{Si}$ \\
Controles individuales & $\mathrm{No}$ & $\mathrm{No}$ & $\mathrm{Si}$ & $\mathrm{Si}$ \\
Control ideología & $\mathrm{No}$ & $\mathrm{No}$ & $\mathrm{No}$ & $\mathrm{Si}$ \\
\hline
\end{tabular}

*** Significatividad al 1\%; ** significatividad al 5\%; ${ }^{*}$ significatividad al $10 \%$.

Notas: La variable alto-ingreso es una variable binaria que toma valor uno para los individuos con valores de la escala de ingresos mayores al p90. Errores estándar clustereados a nivel provincia-año entre paréntesis.

Tabla 5: Efectos heterogéneos: Sector de empleo

\begin{tabular}{lcccc}
\hline & $(\mathrm{I})$ & $(\mathrm{II})$ & $(\mathrm{III})$ & $(\mathrm{IV})$ \\
\hline & & & & \\
Share inmigrantes $(\log )$ & -0.0145 & -0.0117 & -0.00836 & -0.00922 \\
& $(0.0150)$ & $(0.0152)$ & $(0.0152)$ & $(0.0151)$ \\
Share inmigrantes $(\log ) \mathrm{x}$ & $-0.0176^{*}$ & $-0.0174^{*}$ & $-0.0199^{* *}$ & $-0.0208^{* *}$ \\
empleado-privado & $(0.00910)$ & $(0.00910)$ & $(0.00911)$ & $(0.00913)$ \\
& & & & \\
Observaciones & 48,448 & 48,448 & 48,447 & 48,447 \\
R-cuadrado & 0.092 & 0.092 & 0.094 & 0.099 \\
\hline EF país-región-año & $\mathrm{Si}$ & $\mathrm{Si}$ & $\mathrm{Si}$ & $\mathrm{Si}$ \\
Controles provinciales & $\mathrm{No}$ & $\mathrm{Si}$ & $\mathrm{Si}$ & $\mathrm{Si}$ \\
Controles individuales & $\mathrm{No}$ & $\mathrm{No}$ & $\mathrm{Si}$ & $\mathrm{Si}$ \\
Control ideología & $\mathrm{No}$ & $\mathrm{No}$ & $\mathrm{No}$ & $\mathrm{Si}$ \\
\hline
\end{tabular}

*** Significatividad al 1\%; ** significatividad al 5\%; *significatividad al $10 \%$.

Notas: La variable empleado-privado toma valor uno para los empleados privados y por cuenta propia. La muestra se restringe a los ocupados. Errores estándar clustereados a nivel provincia-año entre paréntesis.

que el efecto anti-redistribución es más fuerte en los países más ricos, donde las percepciones erróneas o el prejuicio per se contra los inmigrantes podría ser más fuerte. 
Tabla 6: Efectos heterogéneos: Calificación inmigrantes

\begin{tabular}{lcccc}
\hline & $(\mathrm{I})$ & $(\mathrm{II})$ & $(\mathrm{III})$ & $(\mathrm{IV})$ \\
\hline Share inmigrantes baja-calificación $(\log )$ & $-0.0290^{* *}$ & $-0.0314^{* * *}$ & $-0.0299^{* * *}$ & $-0.0300^{* * *}$ \\
& $(0.0113)$ & $(0.0114)$ & $(0.0113)$ & $(0.0113)$ \\
Share inmigrantes alta-calificación $(\log )$ & 0.00794 & $0.0125^{*}$ & 0.0104 & 0.00937 \\
& $(0.00701)$ & $(0.00753)$ & $(0.00747)$ & $(0.00752)$ \\
& & & & \\
Observaciones & 87,944 & 87,944 & 87,944 & 87,944 \\
R-cuadrado & 0.088 & 0.088 & 0.091 & 0.094 \\
\hline EF país-región-año & $\mathrm{Si}$ & $\mathrm{Si}$ & $\mathrm{Si}$ & $\mathrm{Si}$ \\
Controles provinciales & $\mathrm{No}$ & $\mathrm{Si}$ & $\mathrm{Si}$ & $\mathrm{Si}$ \\
Controles individuales & $\mathrm{No}$ & $\mathrm{No}$ & $\mathrm{Si}$ & $\mathrm{Si}$ \\
Control ideología & $\mathrm{No}$ & $\mathrm{No}$ & $\mathrm{No}$ & $\mathrm{Si}$ \\
\hline
\end{tabular}

*** Significatividad al 1\%; ** significatividad al $5 \%$; ${ }^{*}$ significatividad al $10 \%$.

Notas: Inmigrantes de alta-calificación son aquellos con al menos educación terciaria completa y los de bajacalificación los que tienen terciaria incompleta o menos. Errores estándar clustereados a nivel provincia-año entre paréntesis.

Tabla 7: Efectos heterogéneos: Origen inmigrantes

\begin{tabular}{lcccc}
\hline & $(\mathrm{I})$ & $(\mathrm{II})$ & $(\mathrm{III})$ & $(\mathrm{IV})$ \\
\hline & & & & \\
Share inmigrantes países fronterizos $(\log )$ & $-0.0190^{* * *}$ & $-0.0204^{* * *}$ & $-0.0208^{* * *}$ & $-0.0212^{* * *}$ \\
& $(0.00638)$ & $(0.00636)$ & $(0.00636)$ & $(0.00633)$ \\
Share inmigrantes países no-fronterizos $(\log )$ & -0.00198 & -0.00508 & -0.00530 & -0.00607 \\
& $(0.0112)$ & $(0.0122)$ & $(0.0121)$ & $(0.0121)$ \\
& & & & \\
Observaciones & 84,674 & 84,674 & 84,674 & 84,674 \\
R-cuadrado & 0.089 & 0.089 & 0.091 & 0.094 \\
\hline EF país-región-año & $\mathrm{Si}$ & $\mathrm{Si}$ & $\mathrm{Si}$ & $\mathrm{Si}$ \\
Controles provinciales & $\mathrm{No}$ & $\mathrm{Si}$ & $\mathrm{Si}$ & $\mathrm{Si}$ \\
Controles individuales & $\mathrm{No}$ & $\mathrm{No}$ & $\mathrm{Si}$ & $\mathrm{Si}$ \\
Control ideología & $\mathrm{No}$ & $\mathrm{No}$ & $\mathrm{No}$ & $\mathrm{Si}$ \\
\hline
\end{tabular}

*** Significatividad al 1\%; ** significatividad al $5 \%$; *significatividad al $10 \%$.

Notas: Las observaciones son menos falta de información para algunos países-años. Errores estándar clustereados a nivel provincia-año entre paréntesis.

\subsection{Chequeo de robustez y ejercicio placebo}

Robustez. Como una robustez, exploro los resultados utilizando medidas alternativas para las preferencias por la redistribución. Utilizo preguntas sobre la aceptación de la intervención del Estado en diferentes cuestiones sociales: el bienestar de la población, el empleo y la salud. 
Tabla 8: Efectos heterogéneos: Destino inmigrantes

\begin{tabular}{lcccc}
\hline & $(\mathrm{I})$ & $(\mathrm{II})$ & $(\mathrm{III})$ & $(\mathrm{IV})$ \\
\hline & & & & \\
Share inmigrantes $(\log )$ & -0.0120 & -0.0161 & -0.0155 & -0.0156 \\
& $(0.0131)$ & $(0.0134)$ & $(0.0134)$ & $(0.0133)$ \\
Share inmigrantes (log) x país-rico & $-0.0437^{*}$ & $-0.0390^{*}$ & $-0.0395^{*}$ & $-0.0412^{*}$ \\
& $(0.0233)$ & $(0.0229)$ & $(0.0227)$ & $(0.0226)$ \\
& & & & \\
Observaciones & 87,944 & 87,944 & 87,944 & 87,944 \\
R-cuadrado & 0.088 & 0.088 & 0.091 & 0.094 \\
\hline EF país-región-año & $\mathrm{Si}$ & $\mathrm{Si}$ & $\mathrm{Si}$ & $\mathrm{Si}$ \\
Controles provinciales & $\mathrm{No}$ & $\mathrm{Si}$ & $\mathrm{Si}$ & $\mathrm{Si}$ \\
Controles individuales & $\mathrm{No}$ & $\mathrm{No}$ & $\mathrm{Si}$ & $\mathrm{Si}$ \\
Control ideología & $\mathrm{No}$ & $\mathrm{No}$ & $\mathrm{No}$ & $\mathrm{Si}$ \\
\hline
\end{tabular}

*** Significatividad al 1\%; ** significatividad al 5\%; *significatividad al $10 \%$.

Notas: Los países ricos son los cinco países más ricos en términos de ingreso per cápita en dólares ppp según datos del Banco Mundial (Argentina, Chile, México, Panamá y Uruguay). Errores estándar clustereados a nivel provincia-año entre paréntesis.

También construyo un índice de PCA con las variables de desigualdad, bienestar y empleo. La Tabla 9 muestra que los resultados van en la misma línea que antes. Al analizar el índice de bienestar, el coeficiente alcanza un valor de -0,044. Además, encuentro una mayor correlación al considerar las preferencias de los individuos en cuanto a las intervenciones del gobierno para proporcionar empleo o salud en la economía.

Además, excluyo las ciudades capitales de la muestra para asegurarme de que los resultados no se obtengan de esas ciudades específicas. La Tabla 10 muestra que los resultados permanecen sin cambios después de esta restricción.

Por último, dado que para algunos países sólo hay un censo, restrinjo la muestra a los países que tienen dos censos disponibles. La Tabla 11 muestra que las estimaciones son aún mayores en esta muestra restringida.

Placebo. Para poner a prueba la estrategia empírica hago un ejercicio placebo al realizar las estimaciones sobre una variable de resultado en la que se espera un efecto nulo de la inmigración. Más específicamente, utilizo como variable dependiente el apoyo al aborto que puede ser determinado por valores intrínsecos no relacionados con la inmigración. En la Tabla 12, no encuentro coeficientes estadísticamente significativos en las especificaciones más completas. 
Tabla 9: Robustez: Variables de resultado alternativas

\begin{tabular}{lcccc}
\hline & Índice & Bienestar & Empleo & Salud \\
\hline Share inmigrantes $(\log )$ & $\begin{array}{c}-0.0445^{* * *} \\
(0.0168)\end{array}$ & $\begin{array}{c}-0.0310^{* *} \\
(0.0146)\end{array}$ & $\begin{array}{c}-0.0450^{* * *} \\
(0.0151)\end{array}$ & $-0.0460^{* *}$ \\
& & & & \\
& 52,124 & 52,271 & 52,422 & 35,202 \\
Observaciones & 0.120 & 0.082 & 0.103 & 0.101 \\
R-cuadrado & $\mathrm{Si}$ & $\mathrm{Si}$ & $\mathrm{Si}$ & $\mathrm{Si}$ \\
\hline EF país-región-año & $\mathrm{Si}$ & $\mathrm{Si}$ & $\mathrm{Si}$ & $\mathrm{Si}$ \\
Controles provinciales & $\mathrm{Si}$ & $\mathrm{Si}$ & $\mathrm{Si}$ & $\mathrm{Si}$ \\
Controles individuales & $\mathrm{Si}$ & $\mathrm{Si}$ & $\mathrm{Si}$ & $\mathrm{Si}$ \\
Control ideología & &
\end{tabular}

*** Significatividad al 1\%; ** significatividad al 5\%; *significatividad al $10 \%$.

Notas: Las variables bienestar, empleo y salud se construyen utilizando las respuestas acerca del grado de acuerdo con las siguientes afirmaciones, respectivamente: "El gobierno (país), más que los individuos, debe ser el principal responsable de asegurar el bienestar de la población"; "El gobierno (del país), más que el sector privado, debería ser el principal responsable de la creación de puestos de trabajo."; y "El gobierno (del país), más que el sector privado, debe ser el principal responsable de la prestación de servicios de atención de la salud.”. El índice se construye como el primer componente de un análisis de componente principales con las variables de bienestar, empleo y desigualdad. Las variables dependientes están estandarizadas (z-score). Errores estándar clustereados a nivel provincia-año entre paréntesis

Tabla 10: Robustez: Sin capitales

\begin{tabular}{lcccc}
\hline & $(\mathrm{I})$ & $(\mathrm{II})$ & $(\mathrm{III})$ & $(\mathrm{IV})$ \\
\hline Share inmigrantes $(\mathrm{log})$ & $-0.0209^{*}$ & $-0.0308^{* *}$ & $-0.0298^{* *}$ & $-0.0303^{* *}$ \\
& $(0.0114)$ & $(0.0119)$ & $(0.0119)$ & $(0.0118)$ \\
Observaciones & & & & \\
R-cuadrado & 64,759 & 64,759 & 64,759 & 64,759 \\
\hline EF país-región-año & 0.084 & 0.084 & 0.087 & 0.090 \\
Controles provinciales & $\mathrm{Si}$ & $\mathrm{Si}$ & $\mathrm{Si}$ & $\mathrm{Si}$ \\
Controles individuales & $\mathrm{No}$ & $\mathrm{Si}$ & $\mathrm{Si}$ & $\mathrm{Si}$ \\
Control ideología & $\mathrm{No}$ & $\mathrm{No}$ & $\mathrm{Si}$ & $\mathrm{Si}$ \\
\hline
\end{tabular}

*** Significatividad al 1\%; ** significatividad al 5\%; *significatividad al $10 \%$. 
Tabla 11: Robustness: Two census

\begin{tabular}{lcccc}
\hline & $(\mathrm{I})$ & $(\mathrm{II})$ & $(\mathrm{III})$ & $(\mathrm{IV})$ \\
\hline & & & & \\
Share inmigrantes $(\log )$ & $-0.0411^{*}$ & $-0.0568^{* *}$ & $-0.0560^{* *}$ & $-0.0566^{* *}$ \\
& $(0.0247)$ & $(0.0251)$ & $(0.0250)$ & $(0.0248)$ \\
& & & & \\
Observaciones & 30,009 & 30,009 & 30,009 & 30,009 \\
R-cuadrado & 0.095 & 0.096 & 0.099 & 0.103 \\
\hline EF país-región-año & $\mathrm{Si}$ & $\mathrm{Si}$ & $\mathrm{Si}$ & $\mathrm{Si}$ \\
Controles provinciales & $\mathrm{No}$ & $\mathrm{Si}$ & $\mathrm{Si}$ & $\mathrm{Si}$ \\
Controles individuales & $\mathrm{No}$ & $\mathrm{No}$ & $\mathrm{Si}$ & $\mathrm{Si}$ \\
Control ideología & $\mathrm{No}$ & $\mathrm{No}$ & $\mathrm{No}$ & $\mathrm{Si}$ \\
\hline
\end{tabular}

*** Significatividad al $1 \% ;{ }^{* *}$ significatividad al $5 \% ; *$ significatividad al $10 \%$.

Tabla 12: Placebo: justificación aborto

\begin{tabular}{lcccc}
\hline & $(\mathrm{I})$ & $(\mathrm{II})$ & $(\mathrm{III})$ & $(\mathrm{IV})$ \\
\hline & & & & \\
Share inmigrantes $(\log )$ & 0.0101 & $0.0112^{*}$ & 0.00944 & 0.00950 \\
& $(0.00621)$ & $(0.00673)$ & $(0.00666)$ & $(0.00666)$ \\
& & & & \\
Observaciones & 42,779 & 42,779 & 42,779 & 42,779 \\
R-cuadrado & 0.052 & 0.052 & 0.067 & 0.067 \\
\hline EF país-región-año & $\mathrm{Si}$ & $\mathrm{Si}$ & $\mathrm{Si}$ & $\mathrm{Si}$ \\
Controles provinciales & $\mathrm{No}$ & $\mathrm{Si}$ & $\mathrm{Si}$ & $\mathrm{Si}$ \\
Controles individuales & $\mathrm{No}$ & $\mathrm{No}$ & $\mathrm{Si}$ & $\mathrm{Si}$ \\
Control ideología & $\mathrm{No}$ & $\mathrm{No}$ & $\mathrm{No}$ & $\mathrm{Si}$ \\
\hline
\end{tabular}

*** Significatividad al 1\%; ** significatividad al $5 \%$; *significatividad al $10 \%$.

Notas: La variable dependiente, la justificación del aborto, es una variable binaria que se deriva de la siguiente pregunta: "¿Cree que está justificado interrumpir un embarazo, es decir, abortar, cuando la salud de la madre está en peligro?".

\section{Variaciones de corto plazo en inmigrantes y actitudes}

\subsection{Estrategia empírica}

El análisis de corte-transversal puede sufrir problemas de endogeneidad. Si bien la fuente de sesgo de los "imanes del bienestar" representaría una estimación de "límite inferior", otra heterogeneidad inobservable, como las perspectivas de movilidad ascendente de la provincia, puede amenazar mis resultados. Para intentar abordar este problema potencial, paso a un diseño con efectos fijos que controla los inobservables invariantes en el tiempo a nivel de provincia.

Específicamente, estimo el siguiente modelo de regresión: 


$$
R_{i p c t}=\beta M_{p c t}+X_{i p c t} \theta+Z_{p c t} \phi+\alpha_{p}+\lambda_{c t}+\epsilon_{i p c t}
$$

donde $R_{i p c t}$ es el apoyo a la reducción de las desigualdades de ingreso del individuo $i ; M_{p c t}$ es la proporción de inmigrantes en la la provincia $p$ (en logs); $X_{i p c t}$ es un vector de controles individuales (años de educación, edad, sexo, edad*sexo, religión, estado de empleo, ideología y escala de ingresos) y $Z_{p c t}$ es un vector de controles provinciales (log de la población, tasa de desempleo, media del ingreso per cápita familiar y la proporción de población rural); $\alpha_{p}$ y $\lambda_{c t}$ son efectos fijos de provincia y país-año, respectivamente. Los errores estándar están clustereados al nivel de la provincia para tener en cuenta la potencial correlación serial dentro de las provincias.

Para esta especificación utilizo encuestas de hogares, de modo que para cada onda LAPOP obtengo la proporción de inmigrantes de cada provincia. De esta manera, exploto la variación de corto plazo en los inmigrantes y las actitudes en el tiempo y entre las provincias.

Cabe señalar que, a diferencia de la especificación de corte-transversal que no distingue la dinámica del efecto de la inmigración, el diseño actual se centra en los efectos a corto plazo (alrededor de dos años). Además, este análisis no solo controla por las diferencias temporales entre las provincias que pueden estar relacionadas con las decisiones de inmigración, sino que también controla por el ingreso medio per cápita de la provincia que varía con el tiempo. Por último, esta especificación también pone a prueba la robustez de los resultados ante una fuente alternativa de información para los datos de inmigración.

\subsection{Resultados}

La Tabla 13 muestra las estimaciones del modelo de efectos fijos para el apoyo a la reducción de las diferencias de ingresos (ecuación (4.1)). Para la presentación sigo el mismo esquema que en el análisis corte-transversal. Los resultados muestran de nuevo una clara relación negativa entre la inmigración y las preferencias de redistribución que es estable a través de las especificaciones. La especificación con el conjunto completo de controles muestra que un aumento de un desvío estándar en la proporción de inmigrantes $(1,28)$ reduce el apoyo a las políticas redistributivas en un 9,2\% del desvío estándar de las preferencias. En términos de la variación dentro de la provincia (es decir, en el tiempo), un aumento de un desvío estándar en la proporción de inmigrantes reduce el apoyo a la redistribución en alrededor del 28,2\% del desvío de preferencias dentro de las provincias.

Efectos heterogéneos. Investigo los efectos heterogéneos entre los individuos encuestados y encuentro resultados que son consistentes con los encontrados anteriormente: el efecto antiredistribución es más fuerte para los individuos auto-seleccionados ideológicamente de extrema derecha (Tabla 14), más educados (Tabla 15) y ocupados en el sector privado (Tabla 17) ${ }^{10}$.

En cuanto a los efectos heterogéneos desde el punto de vista de los inmigrantes, la Tabla 18 muestra de nuevo que los resultados provienen exclusivamente de la inmigración de baja calificación.

\footnotetext{
${ }^{10}$ Los resultados también son más fuertes en los encuestados de altos ingresos, aunque no son estadísticamente significativos a los niveles convencionales (Tabla 16).
} 
Tabla 13: Principales resultados: efecto de la inmigración en el apoyo a la reducción de las diferencias de ingresos

\begin{tabular}{lcccc}
\hline & $(\mathrm{I})$ & $(\mathrm{II})$ & $(\mathrm{III})$ & $(\mathrm{IV})$ \\
\hline & & & & \\
Share inmigrantes $(\log )$ & $-0.0736^{* *}$ & $-0.0726^{* *}$ & $-0.0718^{* *}$ & $-0.0719^{* *}$ \\
& $(0.0333)$ & $(0.0333)$ & $(0.0331)$ & $(0.0324)$ \\
Observaciones & 45,918 & 45,918 & 45,918 & 45,918 \\
R-cuadrado & 0.085 & 0.085 & 0.088 & 0.092 \\
\hline EF provincia & $\mathrm{Si}$ & $\mathrm{Si}$ & $\mathrm{Si}$ & $\mathrm{Si}$ \\
EF país-año & $\mathrm{Si}$ & $\mathrm{Si}$ & $\mathrm{Si}$ & $\mathrm{Si}$ \\
Controles provinciales & $\mathrm{No}$ & $\mathrm{Si}$ & $\mathrm{Si}$ & $\mathrm{Si}$ \\
Controles individuales & $\mathrm{No}$ & $\mathrm{No}$ & $\mathrm{Si}$ & $\mathrm{Si}$ \\
Control ideología & $\mathrm{No}$ & $\mathrm{No}$ & $\mathrm{No}$ & $\mathrm{Si}$ \\
\hline
\end{tabular}

*** Significatividad al 1\%; ** significatividad al $5 \%$; significatividad al $10 \%$.

Notas: La variable dependiente mide el apoyo a la reducción de las diferencias de ingresos y se basa en el grado de acuerdo con la siguiente afirmación "El gobierno (país) debe aplicar políticas fuertes para reducir la desigualdad de ingresos entre los ricos y los pobres". Está estandarizada (z-score). Los controles provinciales incluyen el log de la población, la tasa de desempleo, la media del ingreso per cápita familar y la proporción de la población rural; los controles individuales incluyen los años de educación, la edad, el sexo, edad*sexo, la religión, la situación laboral y la escala de ingresos. El control ideológico considera la forma en que el encuestado se sitúa en una escala del 1 al 10 , en la que el 1 es de izquierda y el 10 de derecha. Errores estándar clustereados a nivel provincia entre paréntesis.

Las encuestas de hogares también nos permiten descomponer a los inmigrantes por nivel de ingresos. La Tabla 19 muestra que el efecto anti-distribución proviene principalmente de la inmigración de bajos ingresos. Estos son consistentes, aunque menos pronunciados, con los resultados de nivel de calificación, sugiriendo que los inmigrantes pueden estar sobre-calificados para sus tareas.

\subsection{Robustez y placebo}

Robustez. También considero resultados alternativos ${ }^{11}$. Los resultados son similares a los de la principal variable de resultado, aunque no son estadísticamente significativos, tal vez debido al menor número de observaciones. Además, excluyo las ciudades capitales en la Tabla 21 y los resultados siguen siendo negativos y estadísticamente significativos.

Finalmente, realizo el ejercicio de placebo de usar como variable de resultado las creencias sobre el aborto y encuentro un efecto nulo de la inmigración en esta dimensión (Tabla 22).

\footnotetext{
${ }^{11}$ No uso el resultado de salud considerado en el análisis corte-transversal porque sólo está disponible en una ola de encuestas
} 
Tabla 14: Efectos heterogéneos: Ideología

\begin{tabular}{lcccc}
\hline & $(\mathrm{I})$ & $(\mathrm{II})$ & $(\mathrm{III})$ & $(\mathrm{IV})$ \\
\hline & & & & \\
Share inmigrantes $(\log )$ & -0.0377 & -0.0364 & -0.0356 & -0.0352 \\
& $(0.0352)$ & $(0.0350)$ & $(0.0349)$ & $(0.0341)$ \\
Share inmigrantes (log) x extrema-derecha & $-0.0456^{* *}$ & $-0.0461^{* *}$ & $-0.0461^{* *}$ & $-0.0460^{* *}$ \\
& $(0.0200)$ & $(0.0199)$ & $(0.0199)$ & $(0.0195)$ \\
& & & & \\
Observaciones & 45,918 & 45,918 & 45,918 & 45,918 \\
R-cuadrado & 0.086 & 0.086 & 0.089 & 0.092 \\
\hline EF provincia & $\mathrm{Si}$ & $\mathrm{Si}$ & $\mathrm{Si}$ & $\mathrm{Si}$ \\
EF país-año & $\mathrm{Si}$ & $\mathrm{Si}$ & $\mathrm{Si}$ & $\mathrm{Si}$ \\
Controles provinciales & $\mathrm{No}$ & $\mathrm{Si}$ & $\mathrm{Si}$ & $\mathrm{Si}$ \\
Controles individuales & $\mathrm{No}$ & $\mathrm{No}$ & $\mathrm{Si}$ & $\mathrm{Si}$ \\
Control ideología & $\mathrm{No}$ & $\mathrm{No}$ & $\mathrm{No}$ & $\mathrm{Si}$ \\
\hline
\end{tabular}

*** Significatividad al 1\%; $* *$ significatividad al $5 \%$; significatividad al $10 \%$.

Notas: La variable extrema-derecha es una variable binaria que indica la auto-selección del encuestado en los valores 8,9 o 10 de la escala izquierda-derecha. Errores estándar clustereados a nivel provincia entre paréntesis.

Tabla 15: Efectos heterogéneos: Nivel de calificación

\begin{tabular}{lcccc}
\hline & $(\mathrm{I})$ & $(\mathrm{II})$ & $(\mathrm{III})$ & $(\mathrm{IV})$ \\
\hline & & & & \\
Share inmigrantes $(\log )$ & $-0.0589^{*}$ & $-0.0582^{*}$ & $-0.0600^{*}$ & $-0.0603^{*}$ \\
& $(0.0343)$ & $(0.0342)$ & $(0.0340)$ & $(0.0334)$ \\
Share inmigrantes (log) x alta-calificación & $-0.0274^{* *}$ & $-0.0271^{* *}$ & $-0.0224^{*}$ & $-0.0222^{*}$ \\
& $(0.0123)$ & $(0.0123)$ & $(0.0117)$ & $(0.0117)$ \\
& & & & \\
Observaciones & 45,918 & 45,918 & 45,918 & 45,918 \\
R-cuadrado & 0.085 & 0.085 & 0.088 & 0.092 \\
\hline EF provincia & $\mathrm{Si}$ & $\mathrm{Si}$ & $\mathrm{Si}$ & $\mathrm{Si}$ \\
EF país-año & $\mathrm{Si}$ & $\mathrm{Si}$ & $\mathrm{Si}$ & $\mathrm{Si}$ \\
Controles provinciales & $\mathrm{No}$ & $\mathrm{Si}$ & $\mathrm{Si}$ & $\mathrm{Si}$ \\
Controles individuales & $\mathrm{No}$ & $\mathrm{No}$ & $\mathrm{Si}$ & $\mathrm{Si}$ \\
Control ideología & $\mathrm{No}$ & $\mathrm{No}$ & $\mathrm{No}$ & $\mathrm{Si}$ \\
\hline
\end{tabular}

*** Significatividad al 1\%; ** significatividad al 5\%; *significatividad al $10 \%$.

Notas: La variable alta-calificación identifica individuos con educación terciaria o más. Errores estándar clustereados a nivel provincia entre paréntesis.

\section{El caso de Colombia}

El éxodo venezolano debido a la actual inestabilidad política y a una fuerte crisis económica en el país es un fenómeno bien conocido. Colombia es el primer país destino y varios trabajos han 
Tabla 16: Efectos heterogéneos: Nivel de ingresos

\begin{tabular}{lcccc}
\hline & $(\mathrm{I})$ & $(\mathrm{II})$ & $(\mathrm{III})$ & $(\mathrm{IV})$ \\
\hline & & & & \\
Share inmigrantes $(\log )$ & $-0.0720^{* *}$ & $-0.0710^{* *}$ & $-0.0709^{* *}$ & $-0.0712^{* *}$ \\
& $(0.0333)$ & $(0.0333)$ & $(0.0330)$ & $(0.0323)$ \\
Share inmigrantes $(\log ) \mathrm{x}$ & -0.0233 & -0.0226 & -0.0147 & -0.0128 \\
alto-ingreso & $(0.0262)$ & $(0.0262)$ & $(0.0255)$ & $(0.0249)$ \\
& & & & \\
Observaciones & 45,918 & 45,918 & 45,918 & 45,918 \\
R-cuadrado & 0.085 & 0.085 & 0.088 & 0.092 \\
\hline EF provincia & $\mathrm{Si}$ & $\mathrm{Si}$ & $\mathrm{Si}$ & $\mathrm{Si}$ \\
EF país-año & $\mathrm{Si}$ & $\mathrm{Si}$ & $\mathrm{Si}$ & $\mathrm{Si}$ \\
Controles provinciales & $\mathrm{No}$ & $\mathrm{Si}$ & $\mathrm{Si}$ & $\mathrm{Si}$ \\
Controles individuales & $\mathrm{No}$ & $\mathrm{No}$ & $\mathrm{Si}$ & $\mathrm{Si}$ \\
Control ideología & $\mathrm{No}$ & $\mathrm{No}$ & $\mathrm{No}$ & $\mathrm{Si}$ \\
\hline
\end{tabular}

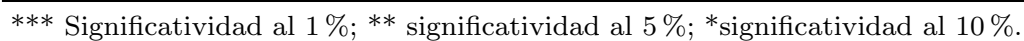

Notas: La variable alto-ingreso es una variable binaria que toma valor uno para los individuos con valores de la escala de ingresos mayores al p90. Errores estándar clustereados a nivel provincia entre paréntesis.

Tabla 17: Efectos heterogéneos: Sector Empleo

\begin{tabular}{lcccc}
\hline & $(\mathrm{I})$ & $(\mathrm{II})$ & $(\mathrm{III})$ & $(\mathrm{IV})$ \\
\hline & & & & \\
Share inmigrantes $(\log )$ & $-0.0762^{* *}$ & $-0.0775^{* *}$ & $-0.0743^{* *}$ & $-0.0733^{* *}$ \\
& $(0.0334)$ & $(0.0334)$ & $(0.0332)$ & $(0.0327)$ \\
Share inmigrantes $(\log ) \mathrm{x}$ & $-0.0316^{* * *}$ & $-0.0318^{* * *}$ & $-0.0355^{* * *}$ & $-0.0344^{* * *}$ \\
empleado-privado & $(0.0119)$ & $(0.0118)$ & $(0.0117)$ & $(0.0115)$ \\
& & & & \\
Observaciones & 25,702 & 25,702 & 25,702 & 25,702 \\
R-cuadrado & 0.093 & 0.093 & 0.097 & 0.102 \\
\hline EF provincia & $\mathrm{Si}$ & $\mathrm{Si}$ & $\mathrm{Si}$ & $\mathrm{Si}$ \\
EF país-año & $\mathrm{Si}$ & $\mathrm{Si}$ & $\mathrm{Si}$ & $\mathrm{Si}$ \\
Controles provinciales & $\mathrm{No}$ & $\mathrm{Si}$ & $\mathrm{Si}$ & $\mathrm{Si}$ \\
Controles individuales & $\mathrm{No}$ & $\mathrm{No}$ & $\mathrm{Si}$ & $\mathrm{Si}$ \\
Control ideología & $\mathrm{No}$ & $\mathrm{No}$ & $\mathrm{No}$ & $\mathrm{Si}$ \\
\hline
\end{tabular}

*** Significatividad al 1\%; ** significatividad al 5\%; *significatividad al $10 \%$.

Notas: La variable empleado-privado toma valor uno para los empleados privados y por cuenta propia. La muestra se restringe a los ocupados. Errores estándar clustereados a nivel provincia entre paréntesis. 
Tabla 18: Efectos heterogéneos: Calificación inmigrantes

\begin{tabular}{lcccc}
\hline & $(\mathrm{I})$ & $(\mathrm{II})$ & $(\mathrm{III})$ & $(\mathrm{IV})$ \\
\hline & & & & \\
Share inmigrantes baja-calificación $(\log )$ & $-0.0605^{* *}$ & $-0.0594^{* *}$ & $-0.0578^{* *}$ & $-0.0589^{* *}$ \\
& $(0.0253)$ & $(0.0252)$ & $(0.0251)$ & $(0.0244)$ \\
Share inmigrantes alta-calificación $(\log )$ & -0.00122 & -0.00101 & -0.000771 & -0.000933 \\
& $(0.00590)$ & $(0.00585)$ & $(0.00587)$ & $(0.00567)$ \\
& & & & \\
Observaciones & 45,918 & 45,918 & 45,918 & 45,918 \\
R-cuadrado & 0.085 & 0.085 & 0.088 & 0.092 \\
\hline EF provincia & $\mathrm{Si}$ & $\mathrm{Si}$ & $\mathrm{Si}$ & $\mathrm{Si}$ \\
EF país-año & $\mathrm{Si}$ & $\mathrm{Si}$ & $\mathrm{Si}$ & $\mathrm{Si}$ \\
Controles provinciales & $\mathrm{No}$ & $\mathrm{Si}$ & $\mathrm{Si}$ & $\mathrm{Si}$ \\
Controles individuales & $\mathrm{No}$ & $\mathrm{No}$ & $\mathrm{Si}$ & $\mathrm{Si}$ \\
Control ideología & $\mathrm{No}$ & $\mathrm{No}$ & $\mathrm{No}$ & $\mathrm{Si}$ \\
\hline
\end{tabular}

*** Significatividad al 1\%;** significatividad al 5\%; *significatividad al $10 \%$.

Notas: Inmigrantes de alta-calificación son aquellos con al menos educación terciaria completa y los de bajacalificación los que tienen terciaria incompleta o menos. Errores estándar clustereados a nivel provincia entre paréntesis.

Tabla 19: Efectos heterogéneos: Nivel de ingreso inmigrantes

\begin{tabular}{lcccc}
\hline & $(\mathrm{I})$ & $(\mathrm{II})$ & $(\mathrm{III})$ & $(\mathrm{IV})$ \\
\hline & & & & \\
Share inmigrantes bajo-ingreso $(\log )$ & $-0.0202^{*}$ & $-0.0206^{*}$ & $-0.0202^{*}$ & $-0.0207^{*}$ \\
& $(0.0119)$ & $(0.0119)$ & $(0.0118)$ & $(0.0113)$ \\
Share inmigrantes alto-ingreso $(\mathrm{log})$ & -0.0104 & -0.0115 & -0.0120 & -0.0113 \\
& $(0.0146)$ & $(0.0150)$ & $(0.0150)$ & $(0.0147)$ \\
& & & & \\
Observaciones & 45,918 & 45,918 & 45,918 & 45,918 \\
R-cuadrado & 0.084 & 0.085 & 0.088 & 0.092 \\
\hline EF provincia & $\mathrm{Si}$ & $\mathrm{Si}$ & $\mathrm{Si}$ & $\mathrm{Si}$ \\
EF país-año & $\mathrm{Si}$ & $\mathrm{Si}$ & $\mathrm{Si}$ & $\mathrm{Si}$ \\
Controles provinciales & $\mathrm{No}$ & $\mathrm{Si}$ & $\mathrm{Si}$ & $\mathrm{Si}$ \\
Controles individuales & $\mathrm{No}$ & $\mathrm{No}$ & $\mathrm{Si}$ & $\mathrm{Si}$ \\
Control ideología & $\mathrm{No}$ & $\mathrm{No}$ & $\mathrm{No}$ & $\mathrm{Si}$ \\
\hline
\end{tabular}

*** Significatividad al 1\%; ** significatividad al $5 \%$; *significatividad al $10 \%$.

Notes: Inmigrantes de alto-ingreso pertenecen a los deciles 8-10. Errores estándar clustereados a nivel provincia entre paréntesis.

estudiado el impacto en diferentes resultados laborales, sanitarios y políticos(ver, for example, Caruso et al., 2019; Rozo y Vargas, 2019). En esta sección analizo si la relación negativa entre la inmigración y el apoyo a las políticas redistributivas se mantiene en el caso específico de los 
Tabla 20: Robustez: Variables de resultado alternativas

\begin{tabular}{lccc}
\hline & Índice & Bienestar & Empleo \\
\hline & & & \\
Share inmigrantes $(\log )$ & -0.0693 & -0.0271 & -0.0543 \\
& $(0.0636)$ & $(0.0367)$ & $(0.0427)$ \\
& & & \\
Observaciones & 27,617 & 27,690 & 27,774 \\
R-cuadrado & 0.129 & 0.094 & 0.107 \\
\hline EF provincia & $\mathrm{Si}$ & $\mathrm{Si}$ & $\mathrm{Si}$ \\
EF país-año & $\mathrm{Si}$ & $\mathrm{Si}$ & $\mathrm{Si}$ \\
Controles provinciales & $\mathrm{Si}$ & $\mathrm{Si}$ & $\mathrm{Si}$ \\
Controles individuales & $\mathrm{Si}$ & $\mathrm{Si}$ & $\mathrm{Si}$ \\
Control ideología & $\mathrm{Si}$ & $\mathrm{Si}$ & $\mathrm{Si}$ \\
\hline
\end{tabular}

*** Significatividad al $1 \% ; * *$ significatividad al $5 \% ; *$ significatividad al $10 \%$.

Tabla 21: Robustez: Sin capitales

\begin{tabular}{lcccc}
\hline & $(\mathrm{I})$ & $(\mathrm{II})$ & $(\mathrm{III})$ & $(\mathrm{IV})$ \\
\hline & & & & \\
Share inmigrantes (log) & $-0.0529^{*}$ & $-0.0510^{*}$ & $-0.0502^{*}$ & $-0.0507^{*}$ \\
& $(0.0299)$ & $(0.0298)$ & $(0.0296)$ & $(0.0292)$ \\
& & & & \\
Observaciones & 33,024 & 33,024 & 33,023 & 33,023 \\
R-cuadrado & 0.080 & 0.081 & 0.084 & 0.087 \\
\hline EF provincia & $\mathrm{Si}$ & $\mathrm{Si}$ & $\mathrm{Si}$ & $\mathrm{Si}$ \\
EF país-año & $\mathrm{Si}$ & $\mathrm{Si}$ & $\mathrm{Si}$ & $\mathrm{Si}$ \\
Controles provinciales & $\mathrm{No}$ & $\mathrm{Si}$ & $\mathrm{Si}$ & $\mathrm{Si}$ \\
Controles individuales & $\mathrm{No}$ & $\mathrm{No}$ & $\mathrm{Si}$ & $\mathrm{Si}$ \\
Control ideología & $\mathrm{No}$ & $\mathrm{No}$ & $\mathrm{No}$ & $\mathrm{Si}$ \\
\hline
\end{tabular}

*** Significatividad al 1\%; ** significatividad al 5\%; *significatividad al $10 \%$.

venezolanos en Colombia.

Utilizo los flujos de inmigración a nivel provincial que provienen del Departamento de Inmigración de Colombia. Estos valores representan los flujos anuales auto-declarados a la provincia (es decir, no se si los individuos se establecieron realmente en la provincia declarada). Además, las encuestas LAPOP para Colombia están disponibles para los años 2012, 2013, 2014, 2016 y 2018. Teniendo esto en cuenta, estimo la ecuación (4.1) para Colombia con esta fuente de datos de inmigración. Cabe notar que, dado que la inmigración fue resultado principalmente de la crisis socio-económica de Venezuela y que se concentró en gran medida en la frontera (es decir, 
Tabla 22: Placebo

\begin{tabular}{lcccc}
\hline & $(\mathrm{I})$ & $(\mathrm{II})$ & $(\mathrm{III})$ & $(\mathrm{IV})$ \\
\hline & & & & \\
Share inmigrantes $(\log )$ & 0.00994 & 0.00674 & 0.00809 & 0.00783 \\
& $(0.0239)$ & $(0.0210)$ & $(0.0205)$ & $(0.0201)$ \\
& & & & \\
Observaciones & 21,553 & 21,553 & 21,553 & 21,553 \\
R-cuadrado & 0.066 & 0.068 & 0.082 & 0.083 \\
EF provincia & $\mathrm{Si}$ & $\mathrm{Si}$ & $\mathrm{Si}$ & $\mathrm{Si}$ \\
EF país-año & $\mathrm{Si}$ & $\mathrm{Si}$ & $\mathrm{Si}$ & $\mathrm{Si}$ \\
Controles provinciales & $\mathrm{No}$ & $\mathrm{Si}$ & $\mathrm{Si}$ & $\mathrm{Si}$ \\
Controles individuales & $\mathrm{No}$ & $\mathrm{No}$ & $\mathrm{Si}$ & $\mathrm{Si}$ \\
Control ideología & $\mathrm{No}$ & $\mathrm{No}$ & $\mathrm{No}$ & $\mathrm{Si}$ \\
\hline
\end{tabular}

*** Significatividad al 1\%; ** significatividad al 5\%; *significatividad al $10 \%$.

en la proximidad), es más plausible sostener el supuesto de exogeneidad.

La Tabla 23 muestra que la inmigración venezolana tiene una clara relación con el apoyo a las políticas de redistribución a través de las especificaciones. Para la lista completa de controles obtengo un coeficiente de -0,09 desvíos estándar. Estos resultados son aún más evidencia del efecto anti-redistribución de la inmigración en la región y pueden explicar el cambio a las ideologías de derecha encontrado por Rozo y Vargas (2019).

Tabla 23: Inmigración y preferencias por la redistribución en Colombia

\begin{tabular}{lcccc}
\hline & $(\mathrm{I})$ & $(\mathrm{II})$ & $(\mathrm{III})$ & $(\mathrm{IV})$ \\
\hline Share inmigrantes $(\log )$ & $\begin{array}{c}-0.0600^{* * *} \\
(0.0200)\end{array}$ & $\begin{array}{c}-0.0983^{* * *} \\
(0.0242)\end{array}$ & $\begin{array}{c}-0.0962^{* * *} \\
(0.0229)\end{array}$ & $\begin{array}{c}-0.0889^{* *} \\
(0.0223)\end{array}$ \\
Observaciones & 5,193 & 5,193 & 5,193 & 5,193 \\
R-cuadrado & 0.030 & 0.031 & 0.041 & 0.045 \\
\hline EF provincia & $\mathrm{Si}$ & $\mathrm{Si}$ & $\mathrm{Si}$ & $\mathrm{Si}$ \\
EF país-año & $\mathrm{Si}$ & $\mathrm{Si}$ & $\mathrm{Si}$ & $\mathrm{Si}$ \\
Controles provinciales & $\mathrm{No}$ & $\mathrm{Si}$ & $\mathrm{Si}$ & $\mathrm{Si}$ \\
Controles individuales & $\mathrm{No}$ & $\mathrm{No}$ & $\mathrm{Si}$ & $\mathrm{Si}$ \\
Control ideología & $\mathrm{No}$ & $\mathrm{No}$ & $\mathrm{No}$ & $\mathrm{Si}$ \\
\hline
\end{tabular}

*** Significatividad al 1\%; ** significatividad al 5\%; *significatividad al $10 \%$. 


\section{Conclusiones}

El debate sobre los inmigrantes está en el centro del debate político global y América Latina no es ajena a él. Según el Informe de Migración Internacional de la ONU de 2017, América Latina y el Caribe (ALC) representa casi el $15 \%$ de la población mundial de inmigrantes. Después de los Estados Unidos, la región es el segundo destino más importante de esta migración y con una tendencia creciente. Por ejemplo, en América del Sur, el número de migrantes intra-regionales aumentó en un $11 \%$ entre 2010 y 2015 (International Organization for Migration, 2017).

Estas tendencias se producen en un contexto de menor apoyo a políticas redistributivas (Figura A1), combinado con una disminución significativa de la tasa de disminución de la desigualdad en la región (Gasparini et al., 2016).

En este trabajo encontré evidencia de que la inmigración puede estar, en efecto, dando forma a las preferencias de redistribución en los países receptores en América Latina. Seguí dos estrategias empíricas: un análisis de corte-transversal con censos armonizados y un enfoque de efectos fijos con encuestas de hogares, ambos unidos con datos actitudinales para el período 2008-2016. Con ambos enfoques encontré una relación negativa y significativa entre la proporción de inmigrantes en una provincia y el apoyo a las políticas redistributivas de la población residente. Este efecto anti-redistributivo es mayor entre ciertos grupos: personas auto-declaradas de extrema derecha, personas altamente calificadas y de altos ingresos y empleados privados. Además, al considerar heterogeneidades por el lado de los inmigrantes, encontré que el efecto se explica plenamente por la inmigración de países fronterizos y de baja calificación. Estos resultados son robustos a medidas alternativas de preferencias de redistribución y un ejercicio de placebo. Finalmente, obtuve resultados confirmatorios para el caso de Colombia.

Dada la relativa similitud de los antecedentes culturales entre los inmigrantes y la población local en el caso de la inmigración de América Latina, cabía esperar un efecto menor o nulo en las respuestas de actitud. Sin embargo, mis resultados pueden estar sugiriendo que la barrera en la extensión de los lazos de solidaridad puede ser simplemente la nacionalidad. 


\section{Referencias}

Alesina, A., Murard, E., y Rapoport, H. (2019). Immigration and preferences for redistribution in europe. Technical report, National Bureau of Economic Research.

Alesina, A. y Stantcheva, S. (2020). Diversity, immigration, and redistribution. AEA Papers and Proceedings, 110:329-34.

Altonji, J. G. y Card, D. (1991). The effects of immigration on the labor market outcomes of less-skilled natives. In Immigration, trade, and the labor market, pages 201-234. University of Chicago Press.

Barone, G., D'Ignazio, A., de Blasio, G., y Naticchioni, P. (2016). Mr. rossi, mr. hu and politics. the role of immigration in shaping natives' voting behavior. Journal of Public Economics, 136:1-13.

Becker, S. O., Fetzer, T., et al. (2016). Does migration cause extreme voting? Center for Competitive Advantage in the Global Economy and The Economic \& Social Research Council, pages $1-54$.

Borjas, G. J. (1999). Immigration and welfare magnets. Journal of labor economics, 17(4):607637.

Brunner, B. y Kuhn, A. (2018). Immigration, cultural distance and natives' attitudes towards immigrants: Evidence from swiss voting results. Kyklos, 71(1):28-58.

Caruso, G., Canon, C. G., y Mueller, V. (2019). Spillover effects of the Venezuelan crisis: migration impacts in Colombia. Oxford Economic Papers. gpz072.

Dahlberg, M., Edmark, K., y Lundqvist, H. (2012). Ethnic diversity and preferences for redistribution. Journal of Political Economy, 120(1):41-76.

Dinas, E., Matakos, K., Xefteris, D., y Hangartner, D. (2019). Waking up the golden dawn: does exposure to the refugee crisis increase support for extreme-right parties? Political analysis, $27(2): 244-254$.

Dustmann, C., Vasiljeva, K., y Piil Damm, A. (2019). Refugee migration and electoral outcomes. The Review of Economic Studies, 86(5):2035-2091.

Edo, A., Giesing, Y., Öztunc, J., y Poutvaara, P. (2019). Immigration and electoral support for the far-left and the far-right. European Economic Review, 115:99-143.

Gasparini, L., Cruces, G., y Tornarolli, L. (2016). chronicle of a deceleration foretold income inequality in latin america in the 2010s. Revista de economía mundial, 43:25-46.

Halla, M., Wagner, A. F., y Zweimüller, J. (2017). Immigration and voting for the far right. Journal of the European Economic Association, 15(6):1341-1385. 
Harmon, N. A. (2018). Immigration, ethnic diversity, and political outcomes: Evidence from denmark. The Scandinavian Journal of Economics, 120(4):1043-1074.

International Organization for Migration (2017). Migration trends in south america. Technical report, IOM. South American Migration Report No. 1. IOM, South America.

Otto, A. H. y Steinhardt, M. F. (2014). Immigration and election outcomes - evidence from city districts in hamburg. Regional Science and Urban Economics, 45:67-79.

Razin, A., Sadka, E., y Swagel, P. (2002). Tax burden and migration: a political economy theory and evidence. Journal of Public Economics, 85(2):167-190.

Rozo, S. y Vargas, J. F. (2019). Brother or invaders? how crisis-driven migrants shape voting behavior. Available at SSRN: https://ssrn.com/abstract=3401036 or http://dx.doi.org/10.2139/ssrn.3401036.

Sasin, M. J. y McKenzie, D. (2007). Migration, remittances, poverty, and human capital: conceptual and empirical challenges. The World Bank.

Steinmayr, A. (2020). Contact versus exposure: Refugee presence and voting for the far-right. The Review of Economics and Statistics, pages 1-47.

Tabellini, M. (2018). Racial heterogeneity and local government finances: Evidence from the great migration. Harvard Business School BGIE Unit Working Paper, (19-006).

Tabellini, M. (2019). Gifts of the Immigrants, Woes of the Natives: Lessons from the Age of Mass Migration. The Review of Economic Studies, 87(1):454-486. 


\section{Apéndice A}

Figura A1: Preferencias por la redistribución en América Latina

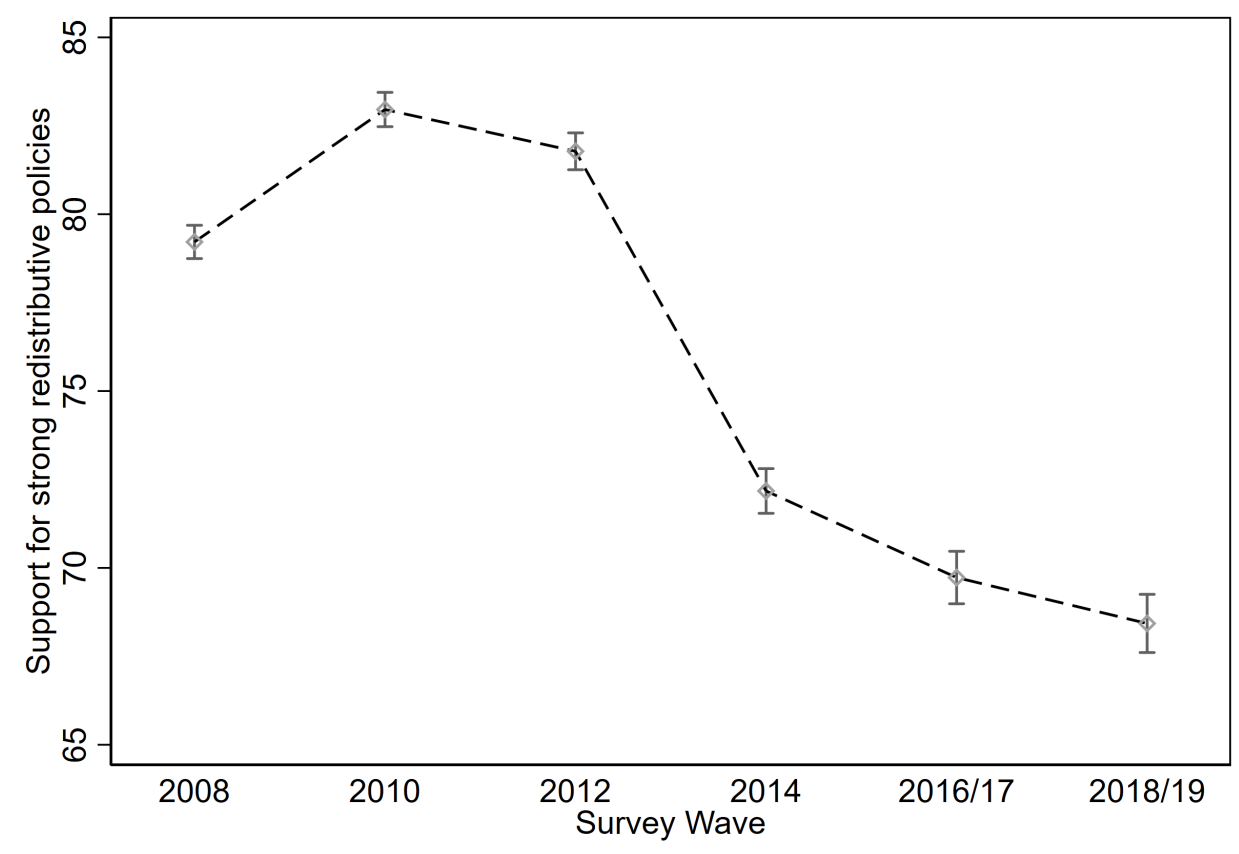

$95 \%$ confidence intervals

Notas: La Figura muestra el porcentaje de individuos que muestran un apoyo significativo a las políticas para reducir la desigualdad de ingresos (valores de 5-7 en la escala de siete puntos en función del acuerdo con la siguiente afirmación : "El gobierno (país) debería aplicar políticas fuertes para reducir la desigualdad de ingresos entre los ricos y los pobres") para cada onda de LAPOP. Los países incluidos son los países latinoamericanos del LAPOP. 


\section{Apéndice B}

Tabla B1: Preguntas de LAPOP para las Preferencias por la Redistribución

\begin{tabular}{|c|c|c|}
\hline Variable & Pregunta & Escala \\
\hline Bienestar & $\begin{array}{l}\text { El gobierno (país), más que los in- } \\
\text { dividuos, debe ser el principal res- } \\
\text { ponsable de asegurar el bienestar } \\
\text { del pueblo. ¿En qué medida está de } \\
\text { acuerdo o en desacuerdo con esta } \\
\text { afirmación? }\end{array}$ & $\begin{array}{l}1 \text { Muy en desacuerdo } \\
\text { - } 7 \text { Muy de acuerdo }\end{array}$ \\
\hline Empleo & $\begin{array}{l}\text { El gobierno (del país), más que el } \\
\text { sector privado, debería ser el prin- } \\
\text { cipal responsable de la creación de } \\
\text { puestos de trabajo. ¿Hasta qué pun- } \\
\text { to está de acuerdo o en desacuerdo } \\
\text { con esta afirmación? }\end{array}$ & $\begin{array}{l}1 \text { Muy en desacuerdo } \\
\text { - } 7 \text { Muy de acuerdo }\end{array}$ \\
\hline Desigualdad & $\begin{array}{l}\text { El gobierno (país) debe aplicar } \\
\text { políticas firmes para reducir la des- } \\
\text { igualdad de ingresos entre los ricos y } \\
\text { los pobres. ¿En qué medida está de } \\
\text { acuerdo o en desacuerdo con esta } \\
\text { afrimación? }\end{array}$ & $\begin{array}{l}1 \text { Muy en desacuerdo } \\
\text { - } 7 \text { Muy de acuerdo }\end{array}$ \\
\hline Salud & $\begin{array}{l}\text { El gobierno (país), más que el sector } \\
\text { privado, debe ser el principal respon- } \\
\text { sable de la prestación de servicios de } \\
\text { atención de la salud. ¿En qué medida } \\
\text { está usted de acuerdo o en desacuer- } \\
\text { do con esta afirmación? }\end{array}$ & $\begin{array}{l}1 \text { Muy en desacuerdo } \\
\text { - } 7 \text { Muy de acuerdo }\end{array}$ \\
\hline
\end{tabular}

La Tabla B2 presenta algunas estadísticas descriptivas de los individuos de la muestra. No hay grandes diferencias entre los países en cuanto a sexo, edad y años de educación. Además, la mayoría de los encuestados están empleados y los estudiantes son generalmente menos del $10 \%$. En cuanto a la afiliación política, la mayoría de los encuestados declaran estar en el centro del espectro político. Sin embargo, hay algunas diferencias entre los países: por ejemplo, en República Dominicana, Colombia y Paraguay hay más gente de derecha que en el promedio de la región, mientras que Uruguay es el país con la mayor proporción de izquierdistas. 
Tabla B2: Estadísticas Descriptivas: Principales variables. LAPOP 2014

\begin{tabular}{|c|c|c|c|c|c|}
\hline País & Hombre & Edad & $\begin{array}{l}\text { Años de } \\
\text { educación }\end{array}$ & Empleado & Estudiante \\
\hline Argentina & $50.9 \%$ & 37.3 & 10.9 & $64.9 \%$ & $7.8 \%$ \\
\hline Bolivia & $50.8 \%$ & 36.4 & 10.4 & $62.8 \%$ & $11.2 \%$ \\
\hline Brasil & $52.0 \%$ & 37.6 & 8.6 & $45.5 \%$ & $3.5 \%$ \\
\hline Chile & $61.2 \%$ & 41.0 & 11.2 & $52.5 \%$ & $6.2 \%$ \\
\hline Colombia & $50.8 \%$ & 35.9 & 9.7 & $57.1 \%$ & $7.0 \%$ \\
\hline Costa Rica & $52.6 \%$ & 38.0 & 8.9 & $51.2 \%$ & $8.3 \%$ \\
\hline Ecuador & $50.8 \%$ & 37.0 & 10.7 & $56.9 \%$ & $7.5 \%$ \\
\hline México & $51.4 \%$ & 37.7 & 9.3 & $52.9 \%$ & $5.1 \%$ \\
\hline Panamá & $50.7 \%$ & 36.8 & 11.0 & $45.6 \%$ & $6.6 \%$ \\
\hline Paraguay & $50.0 \%$ & 36.7 & 9.5 & $59.9 \%$ & $5.1 \%$ \\
\hline Perú & $51.2 \%$ & 36.9 & 11.3 & $56.3 \%$ & $7.1 \%$ \\
\hline República Dominicana & $52.1 \%$ & 37.5 & 9.3 & $50.1 \%$ & $6.9 \%$ \\
\hline Uruguay & $52.1 \%$ & 40.3 & 9.9 & $64.1 \%$ & $4.1 \%$ \\
\hline Total & $52.0 \%$ & 37.6 & 10.0 & $56.3 \%$ & $6.8 \%$ \\
\hline
\end{tabular}

Notas: Elaboración propia con base en datos de LAPOP 2014. 
Tabla B3: Estadísticas Descriptivas: Afiliación política. LAPOP 2014

\begin{tabular}{lccccc}
\hline \hline País & Izquierda & Centro-izquierda & Centro & Centro-derecha & Derecha \\
\hline Argentina & $27.2 \%$ & $7.5 \%$ & $18.1 \%$ & $32.3 \%$ & $15.0 \%$ \\
Bolivia & $19.1 \%$ & $11.1 \%$ & $21.9 \%$ & $33.5 \%$ & $14.4 \%$ \\
Brasil & $21.8 \%$ & $13.2 \%$ & $18.4 \%$ & $22.5 \%$ & $24.0 \%$ \\
Chile & $23.9 \%$ & $12.6 \%$ & $17.7 \%$ & $30.7 \%$ & $15.1 \%$ \\
Colombia & $19.8 \%$ & $10.2 \%$ & $13.9 \%$ & $26.7 \%$ & $29.4 \%$ \\
Costa Rica & $17.7 \%$ & $12.0 \%$ & $14.7 \%$ & $29.2 \%$ & $26.4 \%$ \\
Ecuador & $23.4 \%$ & $14.1 \%$ & $18.5 \%$ & $25.2 \%$ & $18.9 \%$ \\
México & $20.3 \%$ & $12.7 \%$ & $17.4 \%$ & $24.3 \%$ & $25.3 \%$ \\
Panamá & $24.9 \%$ & $13.4 \%$ & $15.4 \%$ & $24.2 \%$ & $22.0 \%$ \\
Paraguay & $17.1 \%$ & $13.1 \%$ & $13.4 \%$ & $28.2 \%$ & $28.3 \%$ \\
Perú & $25.5 \%$ & $10.0 \%$ & $18.2 \%$ & $26.6 \%$ & $19.7 \%$ \\
República Dominicana & $14.3 \%$ & $17.7 \%$ & $13.8 \%$ & $10.0 \%$ & $44.2 \%$ \\
Uruguay & $16.4 \%$ & $17.6 \%$ & $25.8 \%$ & $23.7 \%$ & $16.6 \%$ \\
\hline Total & $\mathbf{2 0 . 9 \%}$ & $\mathbf{1 2 . 6 \%}$ & $\mathbf{1 7 . 8 \%}$ & $\mathbf{2 6 . 2 \%}$ & $\mathbf{2 2 . 5 \%}$ \\
\hline
\end{tabular}

Notas: Elaboración propia con base en datos de LAPOP 2014. Posición ideológica construida en base a una pregunta en la que el encuestado tuvo que situarse en una escala del 1 al 10 en la que el 1 es la izquierda y el 10 es la derecha de la ideología. Considerando esta escala la izquierda comprende 1-2, el centro-izquierda 3-4, el centro 5, el centro-derecha 6-7 y la derecha $8-10$.

La Tabla B4 presenta información sobre los diferentes apoyos a las medidas de redistribución. Como se mencionó anteriormente, estas variables están relacionadas con las preferencias en cuanto a la intervención del gobierno en la provisión de empleo, la salud, la reducción de la desigualdad y la mejora del bienestar de la sociedad. Como se puede observar, dado que la escala de todas las preguntas va de 1 a 7 , existen altos niveles de apoyo a la redistribución en los países analizados. Este apoyo parece ser mayor en el caso de Uruguay, Argentina y Paraguay mientras que, por otro lado, Perú y Bolivia son los países donde, en promedio, hay menos apoyo a este tipo de políticas redistributivas. 
Tabla B4: Estadísticas Descriptivas. Apoyo a la redistribución: LAPOP 2008-2016

\begin{tabular}{lcccc}
\hline \hline País & Bienestar & Empleo & Desigualdad & Salud \\
\hline Argentina & 5.92 & 5.77 & 5.94 & 6.14 \\
Bolivia & 5.44 & 5.63 & 5.30 & 5.74 \\
Brasil & 5.44 & 5.46 & 5.81 & 6.07 \\
Chile & 5.87 & 5.88 & 6.03 & 6.26 \\
Colombia & 5.78 & 6.05 & 5.81 & 6.23 \\
Costa Rica & 5.79 & 5.82 & 5.74 & 6.07 \\
Ecuador & 5.55 & 5.71 & 5.48 & 6.17 \\
México & 5.46 & 5.74 & 5.73 & 6.06 \\
Panamá & 5.57 & 5.48 & 5.52 & 5.95 \\
Paraguay & 6.18 & 6.29 & 5.85 & 6.52 \\
Perú & 5.43 & 5.63 & 5.47 & 5.77 \\
República Dominicana & 5.98 & 6.25 & 6.01 & 6.36 \\
Uruguay & 6.14 & 6.03 & 5.94 & 6.29 \\
\hline
\end{tabular}

Notas: Elaboración propia basada en los datos del LAPOP. 


\section{Apéndice $\mathrm{C}$}

Tabla C1: Datos Inmigración

\begin{tabular}{|c|c|c|}
\hline País & $\begin{array}{c}\text { Censos - } \\
\text { Ipums International }\end{array}$ & Encuestas de Hogares - SEDLAC \\
\hline Argentina & $2001 / 2010$ & 2008/2010/2012/2014/2016 \\
\hline Bolivia & 2001 & - \\
\hline Brasil & $2000 / 2010$ & $2008 / 2010 / 2012 / 2014 / 2016$ \\
\hline Chile & 2002 & $2008 / 2010 / 2012 / 2014 / 2016$ \\
\hline Colombia & 2005 & - \\
\hline Costa Rica & $2000 / 2011$ & $2008 / 2010$ \\
\hline República Dominicana & 2010 & $2008 / 2010 / 2012 / 2014 / 2016$ \\
\hline Ecuador & $2001 / 2010$ & $2008 / 2010 / 2012 / 2014 / 2016$ \\
\hline El Salvador & 2007 & - \\
\hline Guatemala & 2002 & $2008 / 2010 / 2014$ \\
\hline Honduras & 2001 & $2008 / 2010 / 2012 / 2014 / 2016$ \\
\hline México & $2000 / 2010$ & - \\
\hline Nicaragua & 2005 & - \\
\hline Panamá & $2000 / 2010$ & $2008 / 2010 / 2014 / 2016$ \\
\hline Paraguay & 2002 & - \\
\hline Perú & 2007 & $2008 / 2010 / 2012 / 2014 / 2016$ \\
\hline Uruguay & 2011 & $2008 / 2010 / 2012 / 2014 / 2016$ \\
\hline Venezuela & 2001 & - \\
\hline
\end{tabular}

La Tabla C2 muestra que hay una gran variación en la proporción de población de inmigrantes en los países analizados. Mientras que en Argentina, Costa Rica y República Dominicana hay un alto porcentaje de inmigrantes, en Brasil, Colombia y Perú es cercano a cero. Además, un hecho interesante es que estos porcentajes de inmigración están compuestos principalmente por población poco calificada.

Por otra parte, cabe señalar que la variabilidad de la proporción de inmigrantes no es homogénea entre los países. La mayor variabilidad se da en Argentina, Costa Rica y Panamá (desviación estándar superior a dos). Dado que estos países también tienen una elevada proporción de inmigración, es razonable pensar que ésta se concentra en algunas provincias del país. 
Tabla C2: Estadísticas Descriptivas: Inmigración en América Latina 2008-2016

\begin{tabular}{lccc|ccc}
\hline \hline & \multicolumn{3}{c}{ Inmigrantes $\mathbf{( \% )}$} & \multicolumn{3}{|c}{$\begin{array}{c}\text { Baja-calificación } \\
\text { Inmigrantes ( \%) }\end{array}$} \\
\hline País & Media & SD & p50 & Media & SD & p50 \\
\hline Argentina & 5.2 & 4.4 & 5.5 & 4.9 & 4.0 & 5.3 \\
Bolivia & 1.4 & 1.3 & 0.9 & 1.1 & 1.1 & 0.6 \\
Brasil & 0.3 & 0.3 & 0.3 & 0.3 & 0.2 & 0.2 \\
Chile & 1.3 & 0.8 & 1.1 & 1.1 & 0.7 & 1.0 \\
Colombia & 0.2 & 0.1 & 0.2 & 0.2 & 0.1 & 0.2 \\
Costa Rica & 8.8 & 2.2 & 9.6 & 7.7 & 1.9 & 8.4 \\
Ecuador & 1.1 & 0.6 & 0.9 & 0.8 & 0.5 & 0.7 \\
México & 0.7 & 0.7 & 0.6 & 0.5 & 0.5 & 0.4 \\
Panamá & 3.7 & 2.2 & 4.3 & 2.4 & 1.4 & 3.1 \\
Paraguay & 3.4 & 2.0 & 2.1 & 3.0 & 1.9 & 1.8 \\
Perú & 0.3 & 0.2 & 0.2 & 0.2 & 0.1 & 0.1 \\
República Dominicana & 4.0 & 1.9 & 3.7 & 3.7 & 1.9 & 3.2 \\
Uruguay & 2.3 & 1.2 & 2.1 & 2.0 & 0.9 & 1.9 \\
\hline Total & $\mathbf{2 . 3}$ & $\mathbf{2 . 8}$ & $\mathbf{1 . 0}$ & $\mathbf{2 . 0}$ & $\mathbf{2 . 5}$ & $\mathbf{0 . 9}$ \\
\hline
\end{tabular}

Notas: Elaboración propia basada en datos de Impums International. Individuos de baja-calificación son aquellos sin educación terciaria completa. 
Figura C1: Correlación entre los datos de inmigración

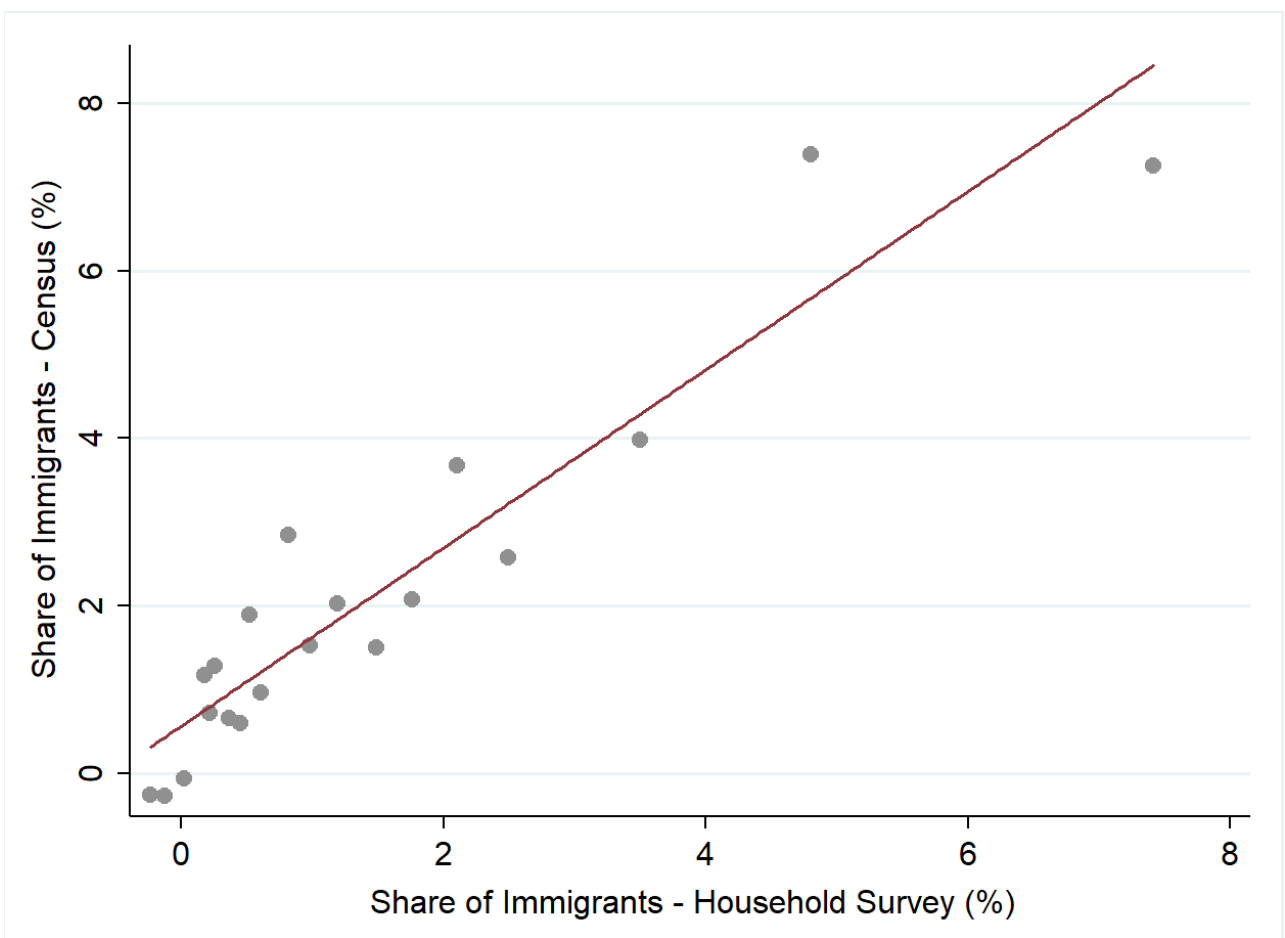

Notas: La Figura muestra el binscatter que se deriva de una regresión del share de inmigrantes de los datos de censo sobre los shares de inmigración provenientes de encuestas de hogares incluyendo efectos fijos por país. La muestra incluye países para los que se dispone de una encuesta de hogares en el año del censo (Argentina, Brasil, Costa Rica y República Dominicana). 